\title{
Effective mixing of laminar flows at a density interface by an integrated ultrasonic transducer $\dagger$
}

\author{
Linda Johansson, Stefan Johansson, Fredrik Nikolajeff and Sara Thorslund*
}

\author{
Received 2nd September 2008, Accepted 8th September 2008 \\ First published as an Advance Article on the web 24th October 2008 \\ DOI: $10.1039 / b 815114 h$
}

\begin{abstract}
An acoustic mixer for glass channel microfluidic systems is presented. An acoustic standing wave, perpendicular to the fluid flow, is generated by the excitation of a miniaturized piezoelectric transducer operated around $10 \mathrm{MHz}$. The transducer is fabricated into a planar printed circuit board structure, constituting the bottom channel wall, which makes the mixer simple to integrate with a wide selection of microfluidic channel designs. The mixing occurs at a fluid-fluid density interface due to the acoustic radiation force; an analytical expression is derived to qualitatively describe this phenomenon. Only a small density difference in the range of $2-5 \%$ is required to achieve $150-270 \%$ peak broadening of a fluorescent sample between sheath flows, which we use as a measure of the mixing efficiency. The mixing efficiency is measured with regard to its sensitivity to the density difference, the fluid velocity and the transducer driving frequency. Transducers at different positions along the microchannel make it possible to compare the mixing of straight versus diagonal flows across the transducer surface. We finally demonstrate enhanced chemical lysis of $E$. coli K12 cells in the device due to active fluid mixing.
\end{abstract}

\section{Introduction}

Microfluidic systems with channel dimensions from a few microns up to several hundreds of microns possess the property of having complete laminar flows, due to a low Reynolds number. Laminar flows are mostly considered advantageous and constitute an important cornerstone within microfluidics, since this makes it possible to predict in a very precise manner where molecules are transported within the system. In the absence of specially designed mixing structures, mixing in these laminarbased flow systems is only accomplished by passive interdiffusion between the fluids. However, many reactions and analyses require well-mixed fluids and to reduce the analysis time of a miniaturized system, the mixing becomes a key unit operation. On-chip applications include e.g. enzyme reactions, cell lysis, immunoassay interactions and hybridization reactions.

The approach in passive mixers is to fabricate structures which use the pumping energy to enhance the interdiffusion effect, whereas active mixers rely on other external energy to achieve mixing by e.g. pressure field-, electrokinetic-, thermal- or acoustic-disturbance. ${ }^{1,2}$ Both passive and active mixing methods have their advantages. Passive mixers do not require external actuators and usually have a more robust and simpler design. Active mixers on the other hand often require less space on the microchip, hence resulting in less dead volumes and shorter performance times for efficient mixing. ${ }^{3}$ Flexibility regarding the flow velocity is an advantage when integrating with other operation steps, making a device compatible with different drive pressure requirements. The ability to turn on and off the

Department of Engineering Sciences, Angström Laboratory, Uppsala University, P. O. Box 534, SE-751 21 Uppsala, Sweden. E-mail: sara. thorslund@angstrom.uu.se; Fax: +4618471 3572; Tel: +46184716805 $\uparrow$ Electronic supplementary information (ESI) available: Figure S1, Figure S2, Figure S3, Movie S4. See DOI: 10.1039/b815114h mixing is an additional feature for active mixers. Several active fluidic mixers based on piezoelectric transducers have been presented. The physical effect that is utilized to cause motion in the fluid may vary, e.g. acoustic streaming, ${ }^{4,5}$ surface acoustic waves, ${ }^{6}$ fluid cavity waves ${ }^{7}$ or oscillating membrane motion. ${ }^{8}$ Beside mixing, the induced fluid motion may also be used for pumping.,9

The system evaluated here utilizes the acoustic radiation force on an interface between two liquids with different density and this interface is oriented parallel to the wave propagation in a standing wave cavity operated around $10 \mathrm{MHz}$ for continuous flow operation. At the operation frequency of $10 \mathrm{MHz}$, viscous absorption losses in the fluid are small and the frequency enables resonance in the fluid. Resonance in the fluid generates high acoustic amplitudes that are useful in employing acoustic forces in general. The lateral dimensions of the transducer $(900 * 900 \mu \mathrm{m})$ enable local activation in a small area of the channel, thereby allowing the handling of small sample volumes with a small dead volume. The PZT $\left(\mathrm{Pb}\left(\mathrm{Zr}_{\mathrm{x}} \mathrm{Ti}_{1-\mathrm{x}}\right) \mathrm{O}_{3}\right)$ transducer can be manufactured into thicknesses with resonance frequencies of around $10 \mathrm{MHz}$ by thick film processing. Some temperature increase is associated with driving a PZT transducer, however limited to a few degrees for a miniaturized transducer. This temperature increase in the fluid above the transducer has been evaluated by our group ${ }^{\mathbf{1 0}}$ and was shown to be in a range acceptable for biological samples without any external cooling of the system.

A theoretical description of the radiation force has earlier been presented by Rozenberg, ${ }^{11}$ however for a density interface perpendicular to the wave propagation direction. In analogy, we have derived an expression for the present system, i.e. where the radiation force acts on an interface parallel to the direction of the wave propagation, between liquids of different density. Surface effects become more pronounced in the micro scale, enabling the utilization of effects that are insignificant in the macro scale. To 
the best of our knowledge, the mixing at a density interface within a microfluidic system has not previously been described. Acoustic mixing by radiation pressure has been presented for microfluidic systems, however, not mentioning any requirements on the fluids to be mixed: Yaralioglu et al. ${ }^{12}$ mixed phenolphthalein (in ethanol) with $0.33 \mathrm{M} \mathrm{NaOH}$ (in ethanol) with a piezoelectric transducer operating at $450 \mathrm{MHz}$ in a $50 \mu \mathrm{m}$ microchannel and Jagannathan et al. ${ }^{13,14}$ mixed two non-labeled fluids with a transducer operating at $400 \mathrm{MHz}$ in a $223 \mu \mathrm{m}$ PDMS channel. Fundamental differences exist between our system and these systems that are operated at 50 times higher driving frequencies: the driving frequency is not matched to a low order of the channel resonance frequency, therefore not benefiting from a strong energy build up in the fluid and the convection of fluid is based on traveling waves rather than standing waves. At a higher driving frequency, energy losses caused by viscous absorption in the fluid become prominent due to a quadratic increase of the absorption coefficient with driving frequency.

In the literature, the mechanism of the described acoustic mixing is not always specified; mixing due to the acoustic streaming and due to the acoustic radiation force can both be described in terms of acoustic radiation pressure. For acoustic streaming usually three types are considered, depending on the length scales they appear on. For travelling waves and acoustic streaming in the direction of the wave propagation direction, Eckart streaming, also known as quartz wind, is a likely candidate $^{15}$ since the inhomogeneities in the acoustic field are larger than the acoustic wavelength. ${ }^{11}$ The driving force, i.e. the difference in the strength of the force between two positions, is a gradient in the time averaged energy density in the direction of the propagating wave. ${ }^{16}$ The gradient is caused by the viscous absorption causing the amplitude to decrease away from the transducer surface and the Eckart streaming is therefore inherently associated with temperature increase in the fluid itself. The absorption coefficient increases quadratically with the driving frequency, ${ }^{11}$ so this effect is mainly observed at higher frequencies. In a standing wave, quartz wind is not observed. ${ }^{17}$ Instead, Rayleigh streaming may occur ${ }^{11}$ and the driving force is also in this case proportional to a spatial gradient in the (time-averaged) energy density. The mechanism is dissipation of acoustic energy adjacent to a solid wall within a resulting boundary layer. ${ }^{18,19}$ Hence, the Rayleigh streaming is also inherently associated with energy loss. In addition to the two kinds of streaming previously mentioned, there also exists Schlichting streaming which occurs within the acoustic boundary layer, i.e. near obstacles in a sound field. ${ }^{11}$ To conclude, the acoustic streaming is a volume effect (though it may occur close to a surface), whereas acoustic radiation force acts on an interface. Most importantly, the acoustic streaming originates from viscous losses, as the origin of the gradient in the acoustic radiation pressure, ${ }^{11,20,21}$ whereas the acoustic radiation forces do not depend on energy losses. ${ }^{22}$

\section{Experimental}

\subsection{Device fabrication}

A schematic view of the platform is shown in Fig. 1a and $1 \mathrm{~b}$. Miniature single layer PZT-ultrasound transducers, $900 \times 900 \times$ $200 \mu \mathrm{m}$, were integrated into the bottom channel wall. The micro transducers were diced out from a $200 \mu \mathrm{m}$ thick commercial piezoelectric Pz-26 plate (Ag-evaporated electrodes, Ferroperm, Kvistgard, Denmark). The transducers were mounted on a printed circuit board (PCB) (1.5 mm thick, $35 \mu \mathrm{m} \mathrm{Cu}$ coating) by conducting silver paint (Electrolube, Derbyshire, UK). Epoxy from Epotec (301-2, Aditya Birla Chemicals, Thailand) was cast around the transducers and the device was cured upside down on a polymer film at $90{ }^{\circ} \mathrm{C}$ for $24 \mathrm{~h}$. The surface was carefully polished with 1200 grit silicon carbide paper, where after through-holes for fluidic connections were drilled in the PCB plate. Finally the top electrode was deposited by evaporating $15 \mathrm{~nm} \mathrm{Cr}$ followed by $150 \mathrm{~nm} \mathrm{Au}$.

The channel-reflector structure was fabricated by wet etching borosilicate glass wafers (Emmaboda Glasteknik, Sweden) to a reflector thickness of $923 \mu \mathrm{m}$ and $71 \mu \mathrm{m}$ channel depth. The etch solution consisted of $\mathrm{HF}: \mathrm{HNO}_{3}$ : DI water $(100: 28: 72, \mathrm{v} / \mathrm{v})$ and $2 \mu \mathrm{m} 1813$ resist (Shipley, Coventry, UK) on $100 \mathrm{~nm} \mathrm{Cr}$ was sufficient as etch mask material. The etch bath was sonicated at repeated occasions in order to achieve even etching. Inlet holes in the glass reflector structure were drilled with a suitable diamond drill; before and after drilling the etched glass structure was heated to $600{ }^{\circ} \mathrm{C}$ and slowly cooled down in order to reduce any a)

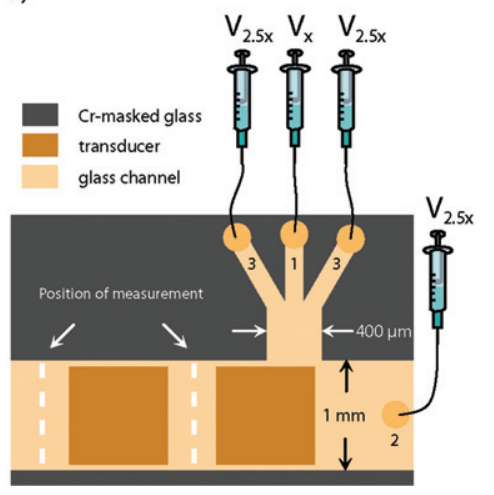

b)

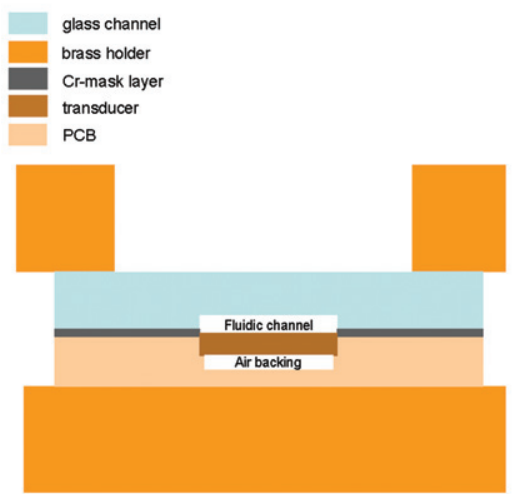

c)

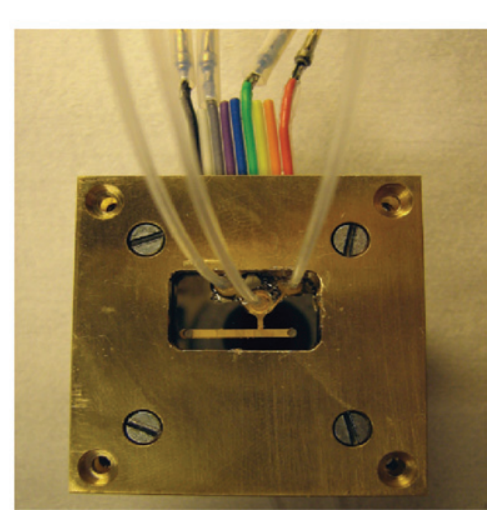

Fig. 1 Top view of the platform showing the glass channel layer with $\mathrm{Cr}$ mask and the underlying PCB layer with integrated transducers and electrical interconnects, (a) schematic (b) cross-sectional view and (c) photo. The channel widths are indicated in the figure and also schematically the positions for the mixing measurements. The cavity was pressure sealed between two tightened brass plates. 
induced stress due to etching and drilling. Fluidic connections on the PCB back side and glass front side were provided by gluing polyethylene tubings of $0.8 \mathrm{~mm}$ inner diameter (Intramedic BD, Sparks, MD) with epoxy. The glass-PCB device was sandwiched between two brass plates and pressure sealed by screwing the upper and lower plates tightly together, see Fig. 1c. Hydrogel applied the glass structure was used to facilitate the sealing between the PCB and the glass channel. It has not been of primary concern to study possible cellular responses due to interactions with the device materials. However, glass is an inert material and any contact between fluid and epoxy or PZT ceramics can be prevented by depositing a Parylene coating by CVD on top of the Au electrode.

The channel was evaluated with four inlet flows and one outlet flow, see Fig. 1a. The primary sample flow (1) with sheath flows (3) entered the cavity through the glass, as seen in the photo in Fig. 1c, while the secondary sample flow (2) and the outlet flow traversed the PCB plate to the backside. One of the transducers was positioned in the t-crossing while the other two were positioned downstream the channel, enabling evaluation of different fluid directions across the transducer surface. Only one transducer was used at the time. The transducer positioned in the t-junction is referred to as the 't-junction transducer' and the second transducer is referred to as the 'mid-channel transducer'.

\subsection{Set-up and quantification}

A common method of evaluating mixing in microchannels is to monitor the color change of a $\mathrm{pH}$-indicator when mixing fluids of different $\mathrm{pH}$-values. ${ }^{3,7,12} \mathrm{We}$ applied the alternative quantification method of registering the position of a fluorescent sample fluid before and after mixing. We used a solution of $0.1 \mathrm{mM}$ Rhodamine B (MP Biomedicals, Eschwege, Germany) in $17 \%$ glycerol (Alfa Aesar, Karlsruhe, Germany)/DI water as the higher-density fluid. DI water was used as the lower-density fluid. The sheath and secondary sample flows contained the same fluid and were both connected to equal sized gastight Hamilton syringes. The primary sample flow contained the fluid with deviating density and was connected to a 2.5 times smaller syringe, see Fig. 1a. A single syringe pump (864, Univentor Limited) was used to drive all syringes simultaneously. The evaluated total volume flow in the main channel was 2.6-85.0 $\mu \mathrm{L} \mathrm{min} \min ^{-1}\left(0.6-20 \mathrm{~mm} \mathrm{~s}^{-1}\right)$ corresponding to a Reynolds number of $0.08-2.5$. The fluorescence was imaged using an inverted Nikon TE-2000-U microscope with a high sensitive cooled CCD camera (SPOT Diagnostic Instruments). The fluorescence intensity profile across the channel at a position $500 \mu \mathrm{m}$ downstream the transducer was plotted using MATLAB 7.1 software. The mixing efficiency was quantified by calculating the percentage broadening of the intensity profile at FWHM (Full-Width Half-Maximum) before and after turning on the ultrasound. The standard deviation was based on three repeated measurements. The transducer was excited by a high-frequency function generator (3320A from Agilent) and an in-house manufactured amplifier. An Agilent 4395A Network Analyzer was used for impedance measurements $(0.22 \mathrm{~V}$ at $50 \Omega)$. The sinusoidal voltage signal at the transducer was $10 \mathrm{~V}_{\mathrm{pp}}$.
The 3D mixing was evaluated by confocal microscopy (Zeiss LSM 510 Meta, $514 \mathrm{~nm}$ laser) for the mid-channel transducer. A $5 \mathrm{x}$ magnification objective (NA of 0.16 ) was used in order to have a large enough working distance due to the brass upper layer and the reflector layer and to have a large enough imaged area to study the entire channel. This low magnification limits the resolution in vertical direction resulting in vertically elongated appearances. This effect was minimized by using a small pinhole. A fluorescent signal was observed from a vertical distance of $110 \mu \mathrm{m}$. To minimize interference effects from the metalized bottom channel surface, this surface was painted dark with a pencil. For the scans with inactivated transducer, a lower amplifier gain in the microscopy software was used in order not to saturate the image at the high concentration of Rhodamine B. For this experiment, the secondary flow was 5 times instead of 2.5 times the sample flow, hence the mixing was not positioned in the middle of the channel. The driving voltage was set to $9 \mathrm{~V}_{\mathrm{pp}}$ and the fluid flow rate was $2.5 \mathrm{~mm} \mathrm{~s}^{-1}$.

\subsection{Mixing demonstration by cell lysis}

We demonstrate the enhanced chemical lysis of E.coli $\mathrm{K} 12$ cells in the device due to fluid mixing of cell suspension and cell lysing solutions. The cell lysing efficiency was compared to when the system is solely based on diffusion-mixing at the fluid interface. Propidium iodide (PI) was used for cell staining, where vital cells with intact cell membranes excluded PI while cells with erupted membranes were readily stained and therefore visible by red-orange fluorescence due to their high intracellular PI concentration. ${ }^{23}$ A cultivated strain of E. coli K12 was initially grown in LB-media at $37^{\circ} \mathrm{C}$ for $24 \mathrm{~h}$. The E. coli suspension was diluted $1: 1$ in PBS-buffer and stained with $5 \mu \mathrm{g} \mathrm{mL} \mathrm{m}^{-1}$ PI. Triton X-100 $\left(20 \%, 1,014 \mathrm{~g} \mathrm{~cm}^{-3}\right)$ in DI-water was used as cell lysing solution and as density interface. The set-up was run with $E$. coli suspension as the primary sample flow $\left(1 \mu \mathrm{L} \mathrm{min}{ }^{-1}\right)$ and Triton $\mathrm{X}-100$ solution as sheath flow. PBS-buffer was used as a second sample flow and the acoustic excitation was performed with the $\mathrm{t}$-junction transducer.

\section{Theoretical model}

The radiation force is most commonly studied when acting upon particles in a fluid. However, the radiation force acts on objects in a broad sense, such as on interfaces between fluids. ${ }^{11,24}$ By convention, the parameter radiation pressure, $P_{r}$, is often used, defined as the time-averaged momentum flux per unit area of an object. ${ }^{11}$ Introducing $P_{r}$ into the Navier-Stokes equation gives

$$
P_{\mathrm{r}}=\langle p\rangle n+\rho_{0}\langle\nu(\nu \cdot n)\rangle-\rho \cdot n
$$

where $n$ is the unit vector normal to the surface, $p$ is the pressure due to the sound field, $v$ is the particle velocity due to sound, $\rho_{0}$ is the density of the medium in the absence of sound and $\sigma$ is the viscous stress tensor $(\sigma=\eta \mathrm{d} u / \mathrm{d} x$, where $\eta$ is the dynamic viscosity). $\sigma$ counteracts the other two terms in equation (3.1) and is proportional to the change in velocity perpendicular to the fluid movement, i.e. the mixing velocity, $u$. For a microfluidic system in general, the viscous forces may be significant relative the pressure or inertial forces. The effect of the viscous 
forces here is a reduction of the fluid mixing motion. Since the mixing velocity depends on the time exposed to the acoustic force, the viscous effect is time dependent. For simplicity this effect will be ignored later on in the simplified model presented.

In a linear expansion of the time-averaged sound pressure, $<p>$, the first order terms do not contribute and including only terms up to the second order gives, ${ }^{11,22,24}$

$$
\langle p\rangle=\frac{\left\langle p_{a}^{2}\right\rangle}{2 \rho_{0} c_{0}^{2}}-\frac{\rho_{0}\left\langle v^{2}\right\rangle}{2}=\left\langle E_{p}\right\rangle-\left\langle E_{k}\right\rangle
$$

where $p_{a}$ is the pressure amplitude due to the acoustic field and $c_{0}$ is the speed of sound. The first term in eqn 3.2 is identified as the potential energy density, $\left\langle E_{p}\right\rangle$, the second term as the kinetic energy density, $\left\langle E_{k}\right\rangle$, and the sum terms (the kinetic energy density with negative sign) is identified as the energy density. Hence the time-averaged sound pressure is identified as the acoustic energy density. Inserting eqn 3.2 into eqn 3.1 and considering that the velocity amplitude is zero in the direction perpendicular to the wave propagation and the surface interface, i.e. the term $v^{*} n$ equals zero, only the first term in eqn (3.2), will give a non-zero contribution in eqn (3.1). For the parallel case we find

$$
P_{r, \text { parallel }}=\frac{\left\langle p_{a}^{2}\right\rangle}{2 \rho_{0} c_{0}^{2}}-\sigma \cdot n
$$

For a standing wave we assume that the initial amplitude can be multiplied by a $Q$-value, to account for several reflections. Since the density and the speed of sound differ for the fluids to be mixed, the acoustic wave will not fulfil the standing wave condition in the cavity to the same degree on both sides of the interface. Assuming that one side of the boundary matches resonance, the other side will display a shift in the position of the node plane and will display lower amplitude in the wave due to mismatch of the standing wave condition. For a density difference of $5 \%$ relative water, the speed of sound changes with $5 \%$ which yields a shift in resonance frequency of $5 \%$. The shift in the position of the node is small but the mismatch in the resonance frequency will have a significant effect. The $5 \%$ mismatch in resonance frequency is in the range of $0.5 \mathrm{MHz}$ for $10 \mathrm{MHz}$ resonance, while for a typical $Q_{S W}$ value of 20-30 half the FWHM value obtained from the real part of the admittance is in the range of $0.15-0.25 \mathrm{MHz}$, and hence the standing wave ratio will be very low in the fluid with miss-matched speed of sound. The interface will be acted upon by a force, $F_{r 1 \rightarrow 2}$, per area, $A$, equal to the change in the radiation pressure across the interface for a standing wave,

$$
\begin{aligned}
\frac{F_{r_{1 \rightarrow 2}}}{A} & =Q_{1} P_{r, \text { parallel }, 2}-Q_{2} P_{r, \text { parallel }, 1} \\
& =\frac{Q_{1}\left\langle p_{a 1}^{2}\right\rangle}{2 \rho_{01} c_{01}^{2}}+\left(-\frac{Q_{2}\left\langle p_{a 2}^{2}\right\rangle}{2 \rho_{02} c_{02}^{2}}\right)-Q_{1} \sigma_{1}\left(1-\frac{Q_{2}}{Q_{1}} \frac{\sigma_{2}}{\sigma_{1}}\right)
\end{aligned}
$$

where $Q_{1}$ is the $Q$-factor for fluid 1 and $Q_{2}$ is the $Q$-factor for the fluid 2. Hereafter the viscous forces will be ignored for simplicity. The $Q$-value of the fluid supporting the standing wave is called $Q_{S W}$, and may be obtained from electrical measurements. The $Q$-value for the non-matched fluid, in this case $Q_{2}$, may be difficult to obtain from a Z-spectra due to coupled resonance modes. The peak-shaped function of $Q$ versus frequency may be approximated by a Gaussian function, using the FWHM value of the standing wave in the channel, as

$$
Q_{2}\left(f_{2}\right)=Q_{S W} e^{\frac{-\left(f_{2}-f_{S W}\right)^{2}}{F W H M^{2}}}
$$

where $f_{S W}$ and $f_{2}$ are design parameters. $f_{S W}$ is the frequency that matches the channel resonance, here for fluid 1 , and is known from mixing efficiency measurements. $f_{2}$ is the frequency for which channel resonance for fluid 2 is obtained. $f_{2}$ is calculated by observing that the speed of sound, and hence the resonance frequency of the fluid layer, changes linearly with the glycerol concentration. ${ }^{25}$ For a given density of fluid 2 the resonance frequency $f_{2}$ is obtained and when operating at the $f_{S W}, Q_{2}$ is found from eqn (3.5) to a value lower than $Q_{S W}$. The pressure amplitude distribution for the two fluids is illustrated in Fig. 2a.

Further, both fluids are excited by the same velocity amplitude, $v_{t}$, at the transducer interface

$\nu_{1}(z=0)=\nu_{2}(z=0)=v_{t}$

If assuming that the kinetic energy, $\left\langle E_{k}\right\rangle$, equals the potential energy, $\left\langle E_{p}\right\rangle$, as is generally expected if considering the entire volume, ${ }^{11}$ the time averaged quadratic pressure amplitude maximum can be expressed in terms of the velocity amplitude, $v_{t}$,

$$
\left\langle p_{a 1}^{2}\right\rangle_{\max }=\rho_{01}^{2} c_{01}^{2}\left\langle v_{t}^{2}\right\rangle \text { and }\left\langle p_{a 2}^{2}\right\rangle_{\max }=\rho_{02}^{2} c_{02}^{2}\left\langle v_{t}^{2}\right\rangle
$$

Hence, the maximal radiation force on an interface parallel to the wave propagation may be expressed as a)

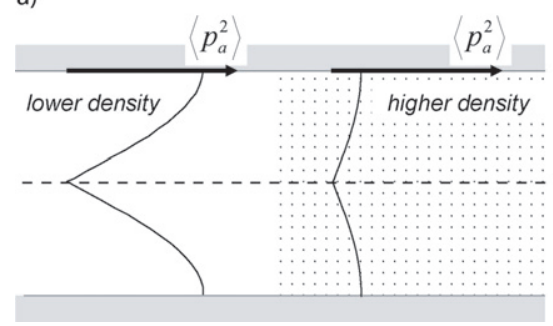

b)

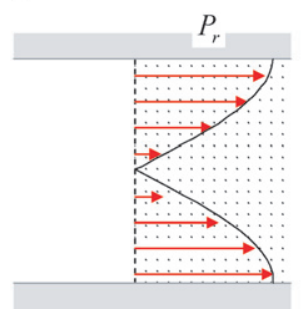

c)

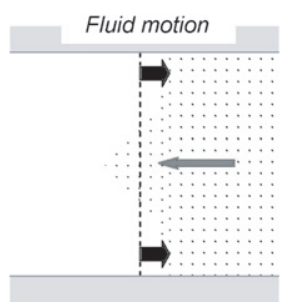

Fig. 2 (a) Illustration of the time-averaged squared pressure amplitude in a standing wave at an interface between a lower-density fluid (white) and a higher-density fluid (grey). (b) The radiation pressure at the interface which is parallel to the wave propagation direction, (c) and the expected movement of the fluids at the interface due to the radiation force (black arrows) and due to conservation of mass flux (grey arrow). 


$$
\begin{aligned}
\left.\frac{F_{r_{1 \rightarrow 2}}}{A}\right|_{\max } & =\frac{Q_{S W}\left\langle p_{a 1}^{2}\right\rangle_{\max }}{2 \rho_{01} c_{01}^{2}}-\frac{Q_{2}\left\langle p_{a 2}^{2}\right\rangle_{\max }}{2 \rho_{02} c_{02}^{2}} \\
& =\frac{\left\langle\left|v_{t}^{2}\right|\right\rangle}{2} Q_{S W}\left(\rho_{01}-e^{\frac{-\left(f_{2}-f_{S W}\right)^{2}}{\frac{F W H M^{2}}{4 \ln 2}}} \rho_{02}\right)
\end{aligned}
$$

for the case where the fluid 1 is supporting the standing wave. Hence, the interface radiation force depends on the excitation amplitude, on the density difference of the fluids and on the $Q_{S W}$-value. For a $Q_{S W}$-value of $20-30$ and a positive density difference of $5 \%$ relative water, the radiation force is positive towards the higher density fluid. The main effect is the build up of amplitude in one of the fluids while the other fluid does not match the standing wave conditions.

For multiple number of interfaces, $i$, of area $A$, in a standing wave in the z-direction between rigid boundaries, the total interface radiation force, here $F_{r}$, can be expressed as

$$
F_{r}(z)=i \cdot A_{\text {int erface }} Q_{S W} \frac{\left\langle\left|v_{t}^{2}\right|\right\rangle}{2} \cos ^{2}\left(\frac{2 \pi}{\lambda / 2} z\right)\left(\rho_{01}-\frac{Q_{2}}{Q_{S W}} \rho_{02}\right)
$$

where $\lambda$ is the wavelength of the acoustic wave. The above expression assumes stepwise density difference at an interface and the force will be reduced as the density difference decreases due to the convective motions caused by the radiation force.

For a standing wave in the channel, Fig. 2a, the top and bottom layers are expected to experience the highest force according to eqn (3.4). The force is directed towards the denser material, Fig. 2b. Due to the conservation of mass flow, a flow in the opposite direction is expected to occur in the middle region where the radiation pressure is weaker, Fig. 2c. According to eqn (3.6), a low-density middle fluid is expected to expand at the upper and lower channel walls. A dense middle fluid on the other hand is expected to expand in the middle of the channel due to mass flow conservation.

The lateral distribution of the acoustic field above a transducer of $550 \mu \mathrm{m}$ length has been measured by light diffraction tomography ${ }^{26}$ and also pictured in the article by Evander et al. ${ }^{10}$ The entire fluid volume above the transducer displays fairly high pressure amplitude and at nine symmetrically displaced positions minimum in pressure amplitude is found. According to eqn (3.6), the interface radiation force is weak at these low pressure amplitude positions.

\section{Measurement results and discussion}

We used both lower- and higher-density middle flow to study the mixing behavior. The significance of the relative position of lower- and higher-density fluids is specifically evaluated in section 4.5. Some of the measurements were performed on the $\mathrm{t}$-junction transducer set-up, and some of the measurements are performed on the mid-channel transducer set-up. Differences between the set-ups were observed and commented on below.

\subsection{Mixing profile and mixing speed}

A typical intensity profile for a set-up with lower-density middle flow and the mid-channel transducer is displayed in Fig. 3a.
The transducer was run with a driving frequency of $10.3 \mathrm{MHz}$ and the system had a total fluid flow of $3 \mu \mathrm{L} \mathrm{min}{ }^{-1}\left(0.7 \mathrm{~mm} \mathrm{~s}^{-1}\right)$. A mixing sequence above the transducer is shown in Fig. $3 b$ with $60 \mathrm{~ms}$ between the images. It is evident that the mixing starts from the interface surfaces and moves the lower-density fluid into the higher-density fluid, in agreement with the proposed theory, provided that the channel is matched for the lower-density fluid. The fluid convection of the lower-density fluid is fastest at four positions on either interface, Fig. 3bii, which corresponds to the spatial pattern of the pressure amplitude in the acoustic near field. At the next stage, Fig. 3biii, the fluid moves in the direction normal to the interface created by the initial mixing movement. The convective motion proceeds until the density difference is equalized, Fig. 3 bvii. Analyzing the mixing speed at a position $500 \mu \mathrm{m}$ downstream the transducer, the main part of the mixing, $80 \%$, occurred

a)

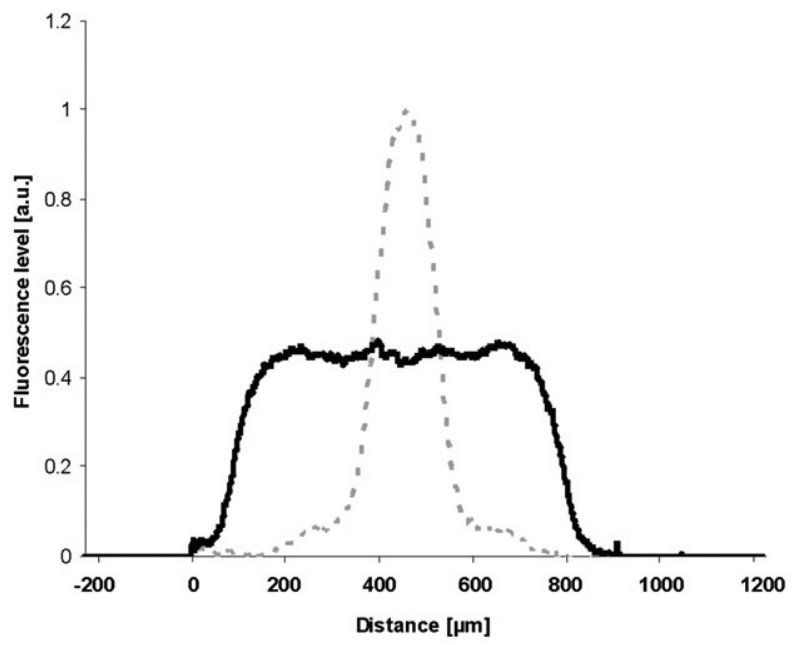

b)

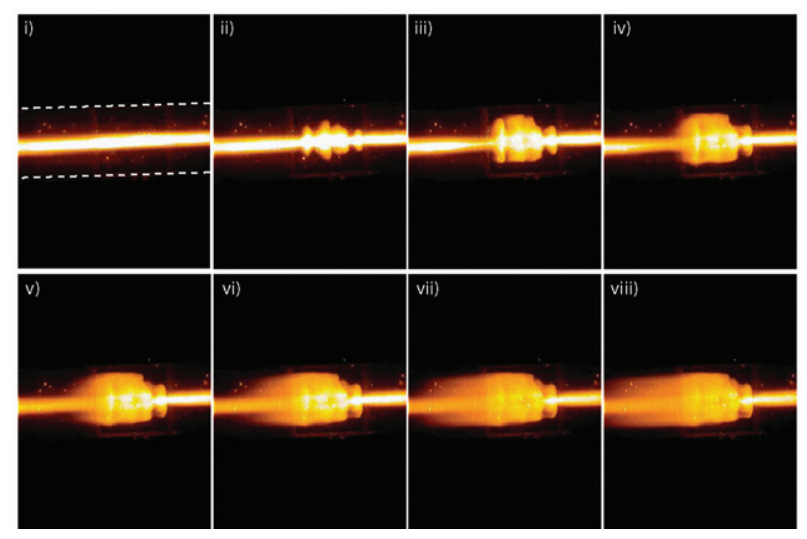

Fig. 3 (a) The intensity profiles across the fluid channel $500 \mu \mathrm{m}$ down stream the midchannel transducer, before (dotted grey) and after (black) transducer activation at a driving frequency of $10.3 \mathrm{MHz}$ and a total fluid velocity of $0.7 \mathrm{~mm} \mathrm{~s}^{-1}$. (b) A sequence of images of the distribution of the fluorescently labeled middle flow above the mid-channel transducer. The flow direction is from right to left. The fluorescent sample flow is of lower density compared to the sheath flow and the total fluid volume has a velocity of $0.7 \mathrm{~mm} \mathrm{~s}^{-1}$. The time interval between each image (i-viii) is $60 \mathrm{~ms}$. 
within $0.36 \mathrm{~s}$ and the mixing was completed after additional $0.72 \mathrm{~s}$.

\subsection{Standing wave cavity}

The standing wave cavity was evaluated by varying the driving frequency for the case of a higher-density middle flow. For the $\mathrm{t}$-junction transducer, the optimal mixing frequency was identified at $10.7 \mathrm{MHz}$. The frequency of this resonance peak coincides satisfactory with simulation results based on an analytical expression ${ }^{27}$ of the fluid-reflector resonance, which predicts a system resonance peak at $10.6 \mathrm{MHz}$ (given sound velocity $_{\text {water }}=1500 \mathrm{~m} \mathrm{~s}^{-1}$, sound velocity reflector $=5530 \mathrm{~m} \mathrm{~s}^{-1}$ and acoustic impedance reflector $=14000 \Omega$ ). A system resonance is expected to yield a high $Q_{S W}$ of the fluid channel. For the midchannel transducer the optimal mixing frequency was identified at $10.3 \mathrm{MHz}$. The prediction of the optimal mixing frequency is complex due to the $3 \mathrm{D}$ characteristics of the acoustic field. Referring to eqn (3.7), the parameters affecting this measurement is the $Q$-value and the fluid excitation amplitude. High excitation amplitude may be found at the series resonance frequency, which is measured by a network analyzer. The series resonance for the mid-channel transducer was found at $10.1 \mathrm{MHz}$, i.e. close to the optimal mixing frequency. The series resonance for the t-junction transducer was found at $9.9 \mathrm{MHz}$.

\subsection{Density sensitivity}

The sensitivity of the mixing efficiency to density difference between the two fluids was evaluated for the t-junction transducer and a higher-density middle flow, by varying the density difference between the two fluids between $0-4.5 \%$, see Fig. $4 a$. At zero density difference no mixing was observed, clearly separating this mixing mechanism from the acoustic mixing observed in a homogenous fluid. Increased mixing strength was observed for increasing density difference. At a density difference of $4.5 \%$, the mixing efficiency was $270 \%$, i.e. a small density difference was sufficient to yield a high mixing efficiency. A curve fit calculated according to eqn (3.7), with a $Q_{S W}$-value of 22 (corresponding to a FWHM value of $0.47 \mathrm{MHz}$ ) obtained from the real part of the admittance is included in Fig. 4a and agrees with the measurements. Some deviations are expected since the mixing versus frequency peak is not perfectly bell-shaped as assumed in theory (see Fig. S3†) and the viscous forces are not included in the model.

\subsection{Volume flow sensitivity}

The sensitivity of the mixing efficiency with fluid velocity is evaluated for the t-junction transducer and a higher-density middle flow. The redistribution of fluorescent sample due to transducer excitation at various flow rates is depicted in the microscopic images in Fig. S1 $\uparrow$. The mixing efficiency decreases with increasing fluid velocity, as seen from the graph in Fig. 4b. The predominating factor for the result is the residence time, i.e. the time the fluid is positioned over the transducer. The graph indicates less mixing at the lowest flow rates (below $1.5 \mathrm{~mm} \mathrm{~s}^{-1}$ ) due to the finite width of the channel and an initially wider sample flow caused by diffusion. The measured mixing efficiency declines at a slower rate with fluid velocity than expected by a)

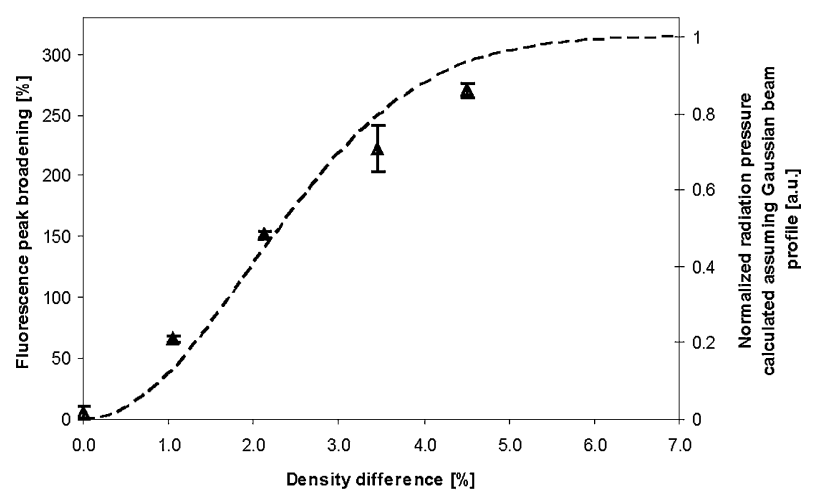

b)

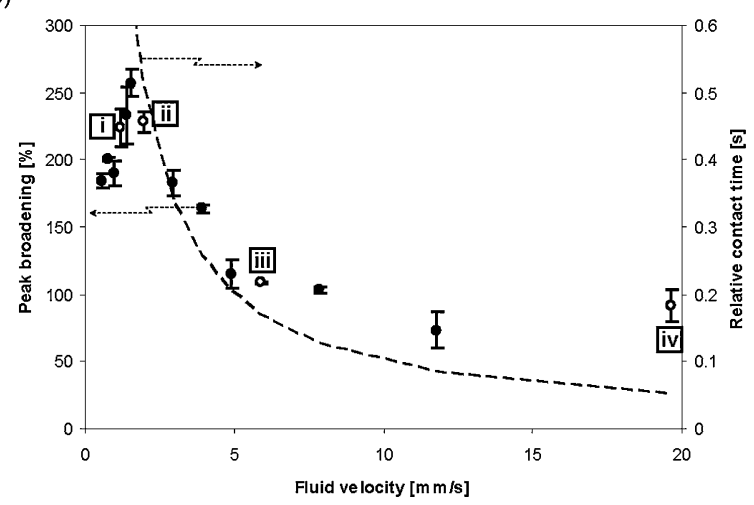

Fig. 4 (a) The measured fluorescence peak broadening (triangles) and the calculated radiation pressure estimation according to equation (3.7) (dotted line), as a function of the relative density difference between the two fluids. The density is assumed to increase linearly with glycerol concentration. ${ }^{25}$ (b) Mixing efficiency measurements and the calculated residence time (dotted line) with increasing total fluid velocities over the transducer. The maximum fluid velocity of $20 \mathrm{~mm} \mathrm{~s}^{-1}$ corresponds to a volume flow of $85 \mu \mathrm{L} \mathrm{min}^{-1}$. The reason for the peak at $1.6 \mathrm{~mm} \mathrm{~s}^{-1}$ is that extended diffusion at lower flows resulted in a smaller relative sample broadening at mixing. For the labeled measurement points (unfilled) (i-iv), images of the sample fluid distribution is available as electronic supplementary information. The standard deviation is based on three repeated measurements.

the residence time model. If the viscous shear forces would be included in the model, their effect would be to counteract the mixing motion, and the mixing efficiency would no longer be constant in time. The viscous shear forces are proportional to the mixing velocity, hence the shear force would be low for short residence times (i.e. high volume flow velocities). At some point in time, the acoustic mixing force and the viscous shear force balance each other and the mixing efficiency is constant. The mixing efficiency would, for the case of high volume flow velocity therefore be higher than expected if not taking the shear forces into account, thereby offering an explanation for the deviation from the residence time curve. Another factor which results in a relatively increased mixing at higher volume flow velocities is the reduction of the acoustic force with time due to depletion of the density difference. In addition, the appearance of the acoustic field at the position of the interface may have an impact on the results. 


\subsection{Relative position of the fluids and 3D evaluation}

The significance of the relative position of the higher- and the lower-density fluid is evaluated for the mid-channel transducer. The mixing efficiency and the process of broadening are both observed to differ depending on whether the middle flow is the lower- or higher-density fluid. For the case of a lower-density middle fluid, pictured in Fig. 5a, the broadening of the middle flow is most efficient, reaching a peak broadening of $400 \%$ (FWHM of $0.65 \mathrm{MHz}$ ) as compared with $270 \%$ (FWHM of $0.55 \mathrm{MHz}$ ) for the case of a higher-density middle flow. The lateral variations in the acoustic pressure field is observed to influence the movement of the fluids: at the points of minimum pressure, slight striations in the fluorescent distributions are observed as darker regions further downstream from the transducer, marked by arrows in Fig. 5ai and 5aii. The movement of the lower-density middle flow seems to occur in multiple layers, as marked by the arrows in the close-up in Fig. 5aii. This is in agreement with the illustration in Fig. 2, where a fluorescent lower-density fluid is expected to move in the top and bottom regions of the microchannel. For the case of a higher-density middle flow, the fluorescent fluid motion is not observed to occur

a)
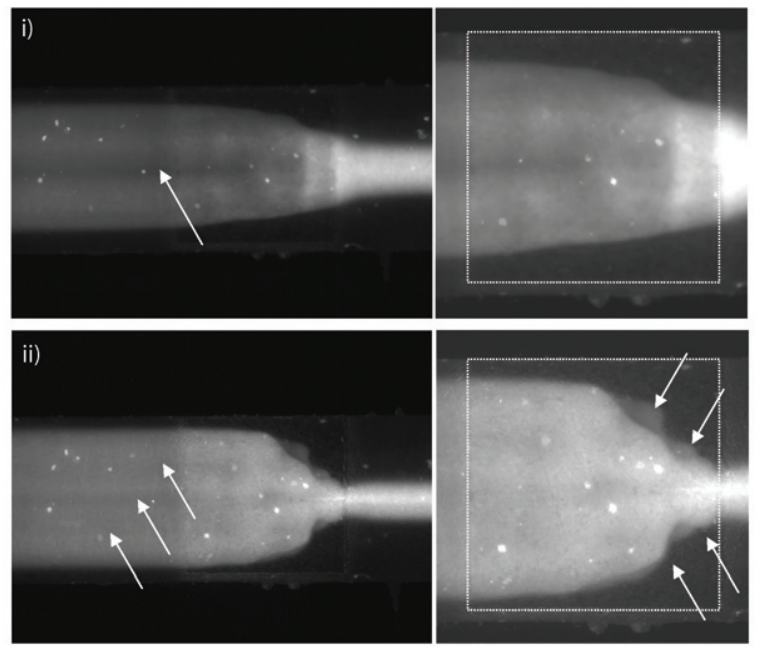

b)

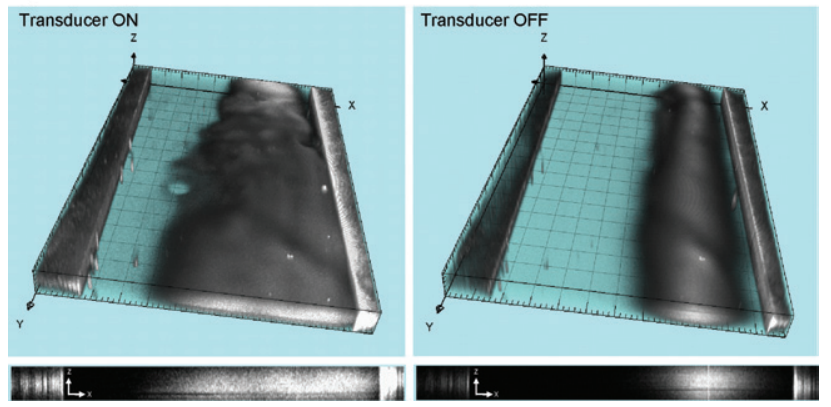

Fig. 5 (a) Mixing at the mid-channel transducer (i) with a higher-density middle flow and (ii) with a lower-density middle flow. Dark striations are observed in (i) and (ii) as marked by arrows. In the close up, the arrows indicate regions where the fluid movement can be observed to occur at multiple layers. (b) Confocal microscopy images showing the mixing process in 3D and a channel cross-section scan at a position $500 \mu \mathrm{m}$ downstream the mid-channel transducer for the transducer $\mathrm{ON}$ and OFF, respectively. in different layers, close-up in Fig. 5ai. The initial movement is smaller and the near field pattern is therefore less visual. Only one single dark striation is formed as opposed to three for the case of lower-density middle flow. This is an agreement with the movement being an indirect effect that is due to the conservation of mass flux.

The 3D mixing confocal microscopy images are shown in Fig. 5b, for the transducer activated and inactivated respectively. Due to the large secondary flow, the mixing area is shifted to one side of the channel. In both images a dark line is observed, probably resulting from the absorbing paint at the channel bottom. As far as can be concluded from theses images, the mixing is three dimensional and does not result in a layered concentration distribution in the vertical direction.

\subsection{Cell lysis}

We investigated if the efficiency of an on-chip chemical cell lysis process could be enhanced by the acoustic mixing step. The set-up included a suspension of $E$. coli as sample flow and Triton X-100 as sheath flows. The cells were initially stained with PI, which is excluded from vital cells with intact cell membranes whereas cells with erupted membranes are stained and visible due to concentrated fluorescence. For an inactivated transducer, the cell and lysing solutions are only mixed by diffusion and few cells along the interface are lysed and PI stained, see Fig. S2 $\uparrow$. The images were captured at the end of the channel, $7 \mathrm{~mm}$ downstream the transducer to allow some time for the cell lysing and staining process. The cell suspension and the lysing reagent mixed at a small density difference between the solutions. The result is an obvious increase in chemical cell lysis as seen from the amount of fluorescent cells. This cell lysis demonstration is only one example of the benefit of an easily integrated mixer; other examples being analysis based on surface-free species interactions, hybridizations or enzyme mediated reactions.

\section{Conclusions}

We present an effective mixing device for microfluidic applications. It is shown that the acoustic radiation force generated by an integrated piezoelectric transducer acts on the fluid density interface parallel to the wave propagation direction. A density difference between the sample and sheath fluids of $4.5 \%$ yields a mixing efficiency of $400 \%$ peak broadening for the case of a lower-density middle flow. For the case of a higher-density middle flow, the mixing efficiency is less, around $250 \%$ peak broadening, in agreement with the proposed theory that this movement of the fluid not supporting a standing wave is an indirect mass-conservation effect. The sensitivity of the mixing efficiency with density difference agrees well with the dependence of density difference derived in a simplified analytical expression. The mixing efficiency declines with increasing fluid velocity but mixing is still evident at $85 \mu \mathrm{L} \mathrm{min}{ }^{-1}$. The acoustic mixing was demonstrated to strongly increase chemical cell lysing upon mixing of an E. coli cell suspension with lysing reagent. This method provides stronger evidence of mixing efficiency than the peak broadening evaluation since it is required that cells are in direct contact with the lysing buffer in order to give the fluorescent signal. 
Advantages of the described device include a small mixing site for the miniaturized transducer, hence resulting in a small dead volume. For this standing wave mixer, no acoustic streaming was observed. Compared with mixing induced by acoustic streaming it is advantageous that the mixing is not caused by acoustic absorption losses, especially when temperature increase in the channel is critical.

The requirements for the described system are to drive at the channel resonance and to have high acoustic energy density ( $Q$-value) in one of the fluids relative to the other fluid, which is obtained by the different fluid densities. For an optimal design, it is also desirable to achieve a large interface area compared to the volume in the mixing zone. It is therefore expected that the mixing would be improved by decreasing the channel width for a constant volume. An increased channel height requires a larger transducer thickness operated at a lower frequency. Since the frequency is not a parameter in eqn (3.7), the radiation force does not increase by operating at a higher (or lower) driving frequency. However, the acoustic field would be altered by changing the ratio of the transducer thickness to the transducer area.

An alternative application of the described mixing method may be to estimate the potential energy in a standing wave cavity, since the evaluation of resonators in sealed microchannels is a practically difficult task.

\section{Acknowledgements}

Stefan Bertilsson is acknowledged for the supply of the E. coli K12 bacteria and Jonathan Bagge is acknowledged for image data extraction. We thank Jonas Bergquist and Zhigang Wu for valuable discussions. We thank Javier Sanchez and Jan Grawé for assistance with the confocal microscopy measurements.

\section{References}

1 N. T. Nguyen and Z. G. Wu, J Micromech Microeng, 2005, 15, R1-R16.

2 V. Hessel, H. Lowe and F. Schonfeld, Chemical Engineering Science 5th International Symposium on Mixing in Industrial Processes (ISMIP5), 2005, 60, 2479-2501.
3 A.-1. Liu, F.-y. He, K. Wang, T. Zhou, Y. Lu and X.-h. Xia, Lab Chip, 2005, 5, 974-978.

4 J. C. Rife, M. I. Bell, J. S. Horwitz, M. N. Kabler, R. C. Y. Auyeung and W. J. Kim, Sensor Actuat a-Phys, 2000, 86, 135-140.

5 T. Kamakura, K. Matsuda, Y. Kumamoto and M. A. Breazeale, J Acoust Soc Am, 1995, 97, 2740-2746.

6 W. K. Tseng, J. L. Lin, W. C. Sung, S. H. Chen and G. B. Lee, $J$ Micromech Microeng, 2006, 16, 539-548.

7 M. Bengtsson and T. Laurell, Anal Bioanal Chem, 2004, 378, 17161721.

8 Z. Yang, S. Matsumoto, H. Goto, M. Matsumoto and R. Maeda, Sensor Actuat a-Phys, 2001, 93, 266-272.

9 H. Y. Yu, J. W. Kwon and E. S. Kim, J Microelectromech S, 2006, 15, $1015-1024$

10 M. Evander, L. Johansson, T. Lilliehorn, J. Piskur, M. Lindvall, S. Johansson, M. Almqvist, T. Laurell and J. Nilsson, Anal Chem, 2007, 79, 2984-2991.

11 L. D. Rozenberg, High-intensity ultrasonic fields, Plenum Press, New York, 1971.

12 G. G. Yaralioglu, I. O. Wygant, T. C. Marentis and B. T. KhuriYakub, Anal Chem, 2004, 76, 3694-3698.

13 H. Jagannathan, G. G. Yaralioglu, A. S. Ergun, F. L. A.-D. Degertekin, F.L. and B. T. A.-K.-Y. Khuri-Yakub, B.T., Ultrasonics Symposium, 2001 IEEE Ultrasonics Symposium, 2001 IEEE VO - 2, 2001.

14 H. Jagannathan, G. G. Yaralioglu, A. S. Ergun, and B. T. A.-K.-Y. Khuri-Yakub, B.T., Micro Electro Mechanical Systems, 2003. MEMS03 Kyoto. IEEE The Sixteenth Annual International Conference on, 2003.

15 A. K. Larisa and W. T. Coakley, The Journal of the Acoustical Society of America, 2004, 116, 1956-1966.

16 H. Mitome, T. Kozuka and T. Tuziuti, Jpn J Appl Phys 1, 1995, 34, 2584-2589.

17 G. Whitworth and W. T. Coakley, J Acoust Soc Am, 1992, 91, 79-85.

18 J. Lighthill, J Sound Vib, 1978, 61, 391-418.

19 L. A. Kuznetsova and W. T. Coakley, Biosensors and Bioelectronics, 2007, 22, 1567-1577.

20 W. P. Mason, and R. N. Thurston, Physical acoustics : principles and methods, Academic P., New York, 1965.

21 J. F. Spengler, W. T. Coakley and K. T. Christensen, Aiche J, 2003, 49, 2773-2782.

22 S. M. Woodside, B. D. Bowen and J. M. Piret, Aiche J, 1997, 43, $1727-1736$

23 H. Lu, M. A. Schmidt and K. F. Jensen, Lab Chip, 2005, 5, 23-29.

24 H. Mitome, Electron Comm Jpn 3, 1998, 81, 1-8.

25 R. H. Perry, C. H. Chilton, and J. H. Perry, Chemical engineers' handbook, 5th edn., McGraw-Hill, New York, 1973.

26 T. Lilliehorn, U. Simu, M. Nilsson, M. Almqvist, T. Stepinski, T. Laurell, J. Nilsson and S. Johansson, Ultrasonics, 2005, 43, 293303.

27 M. Hill, J Acoust Soc Am, 2003, 114, 2654-2661. 